\title{
Diagnóstico de las competencias informacionales en Ciencias de la Información desde la percepción del estudiante de la Universidad de la Habana
}

\author{
Marlery Sánchez Díaz *
}

Artículo recibido:

10 de enero de 2014.

Artículo aceptado:

9 de octubre de 2014.

\section{Resumen}

En la actual sociedad de la información es importante saber acceder, analizar, evaluar y utilizar la información adecuadamente; para ello son necesarias las competencias relacionadas con la búsqueda, la evaluación, el tratamiento y la comunicación de la información. Estas competencias se hacen aún más importantes para los profesionales de la información, pues son quienes deben lograr la correspondencia entre los recursos y los requerimientos de información, lo que implica la identificación de necesidades y recursos, la representación y organización de la información y la satisfacción de los destinatarios. Tomando como referencia el cuestionario ALFIN-HUMASS, en el presente trabajo se estudian la motivación y la autoeficacia en cuanto a las

* Universidad de la Habana, Cuba. infctf@biocen.cu

INVESTIGACIÓN BIBLIOTECOLÓGICA, Vol. 29, Núm.67, septiembre/diciembre, 2015, México, ISSN: 0187-358X. pp. 201-218 
competencias relacionadas con la información de los estudiantes de la titulación de Ciencias de la Información de la Universidad de la Habana, especialmente en cuatro categorías (búsqueda, evaluación, tratamiento y comunicación de información), desde la autoevaluación; se analizan además los entornos de aprendizaje a partir de criterios como la biblioteca, individual o autodidacta, cursos o clases. Se reflexiona acerca de la importancia que dan estos estudiantes a las competencias informacionales y su nivel de destreza, así como también las necesidades de aprendizaje.

Palabras clave: Competencias informacionales; Ciencias de la Información.

\section{Abstract \\ Diagnosis of Information Science Informational litera- cy from the perspective of the student of the University of Havana \\ Marlery Sánchez-Diaz}

The Information Society of today requires students to access, analyze, evaluate and use information properly. To this end, students need to acquire competencies associated with search, evaluation, processing and communication of information. These competencies are especially important in information professionals, who are charged with the task of matching information resources with information needs. This implies the ability to identify needs and resources, and organize and represent information to the satisfaction of users. Using the IL-HUMASS self-assessment questionnaire, students' perceptions of motivation and self-efficacy were studied in terms of the competencies associated with four overarching informational categories: Search, Evaluation, Processing and Communication. Moreover, the study examines the learning contexts of self-guided or independent learning, library work, coursework and classrooms. The study offers a reflection on the degree of importance students lend to informational competencies and their perceived degree of expertise, while also drawing some conclusion about learning needs.

Keywords: Information competencies; Information sciences. 


\section{INTRODUCCIÓN}

Toy, cuando la información se hace determinante para la toma de de1 cisiones y crece vertiginosamente su importancia, un profesional con saber especializado en información se vuelve el actor principal de la sociedad en que vivimos.

El profesional de la información, al constituir el imprescindible puente entre los suministradores y los usuarios de la información, un puente cuyo soporte son las llamadas nuevas tecnologías de información y comunicación, se encuentra en medio del complejo mundo de las competencias [...]. Las cambiantes circunstancias de sus posibles mercados y entornos laborales, también en constante transformación, imponen nuevas y disímiles competencias a los profesionales de la información [...]. Su conocimiento no sólo es esencial para sus instituciones formadoras, desde el punto de vista académico, sino para los propios profesionales [...]. (Sánchez y Vega, 2004: s. pág.)

La sociedad de la información y del conocimiento impone que los profesionales de la información sean competentes en información.

Los bibliotecarios deben tener su propia alfabetización o 'competencia informacional', es decir, dominar los conceptos propios del mundo de la información, sus procedimientos y valores para realizar de modo contextualizado, reflexivo e intencional la selección, evaluación, integración, uso y comunicación ética de la información [...]. Respecto a la competencia informacional propia, aunque deba ser constantemente renovada en el contexto tecnológico y científico actual, normalmente se presupone, al ser inherente al ejercicio profesional [...]. (Gómez Hernández, 2009: 107)

Además, es este profesional quien debe formar competencias informacionales para toda la sociedad, tomando en cuenta que el concepto de formación y aprendizaje a lo largo de la vida, que supone la actualización continua para el desarrollo correcto en el estudio, la profesión, en lo cultural y lo social, hace imprescindibles estas competencias. Este tema se irradia cada vez más en la docencia universitaria ya que los conocimientos, habilidades y actitudes relacionados con la información que se desarrollen durante esta etapa educativa permitirán competir con éxito en el mercado laboral. La educación superior, tal como plantean Pinto, Doucet y Fernández-Ramos (2008), debe responder a esta nueva expectativa, haciendo énfasis en que estas competencias van más allá de la instrucción bibliográfica y del manejo de la tecnología pues incluyen cuestiones analíticas, pensamiento crítico y solución de problemas. 
La carrera de Ciencias de la Información (CI) tiene la tarea de formar egresados capaces de resolver con su actividad la contradicción que se produce entre la accesibilidad y disponibilidad de recursos de información y las necesidades, expresadas o no, de información y de formación de diferentes personas o grupos sociales. Este profesional debe lograr la correspondencia entre los recursos y los requerimientos de información, lo que implica la identificación de necesidades y recursos, la representación y organización de la información y la satisfacción de los destinatarios.

Existe literatura abundante sobre alfabetización informacional en la educación superior; sin embargo, son escasos los trabajos que se ocupan de las cuestiones subjetivas de las competencias informacionales en los estudiantes de CI. En el caso de Cuba, Quindemil (2010) presenta una propuesta educativa que facilita la introducción de la ALFIN en el modelo de continuidad de estudios para la especialidad de CI, en el Plan de Estudios C perfeccionado en la Universidad Agraria de la Habana; por su parte, Meneses-Placeres y Frías-Guzmán (2011) examinan la presencia de los postulados de la ALFIN en la formación del profesional de la información a través del diseño curricular y caracterizan la inserción de la ALFIN en el Plan de Estudios D de CI en la Universidad Central Marta Abreu de Las Villas.

En cuanto a la Universidad de la Habana, se encontró un estudio exploratorio sobre las competencias informacionales de los estudiantes, en el que se incluyó a los de CI (González, Sánchez y Lezcano, 2012) pero no se hace un análisis específico de ellos. Por consiguiente, el objetivo principal de este trabajo es ofrecer un diagnóstico de la impresión que tienen los estudiantes de CI de la Universidad de la Habana sobre su motivación y dominio de las competencias informacionales, así como las vías de adquisición de las mismas.

\section{Marco teóRICO}

Las competencias informacionales consisten en poner en práctica, de forma combinada o integrada, en un contexto y con un contenido determinado (transferible), todos los recursos (habilidades, conocimientos, actitudes) para solucionar con éxito problemas y aprender a aprender, a partir de la interacción efectiva con la información; sin delimitación de tipo, formato y soporte. Son necesarias en cualquier ámbito e incluyen otras competencias como las tecnológicas, las bibliotecarias, las bibliográficas, el pensamiento crítico y las sociales (Sánchez, 2008b). "Con el fin de obtenerlas en grado perfecto y a un nivel de excelencia es preciso gestionarlas [...]. La gestión de competencias en información transcurre por diferentes etapas: identifica- 
ción, normalización, evaluación, desarrollo y certificación" (Sánchez, 2008a: s. pág.).

La identificación y normalización de competencias informacionales se ha llevado a cabo a través de diferentes modelos y normas, tal es el caso de las Normas sobre aptitudes para el acceso y uso de la información en la educación superior de la Association of College and Research Libraries (ACRL/ ALA, 2000). La formación de competencias en la perspectiva informacional consiste en el proceso de enseñanza-aprendizaje que facilita la adquisición de tales competencias al nivel requerido. Las maneras de adquirir las competencias se enmarcan en el sistema formal (formación previa, inicial y básica/ antes de la vida activa y fuera del contexto del trabajo) fuera del currículo o dentro del currículo como asignatura propia o parte de una asignatura; en el sistema no formal (formación continua, ocupacional y continua/durante la vida activa) y en el sistema informal (formación a través de la experiencia, por el ejercicio mismo de una actividad profesional) (Sánchez, 2010b).

La evaluación de competencias informacionales es el proceso de verificación de evidencias acerca del desempeño relacionado con la información de un individuo contra el estándar definido en la norma. Esta puede ser diagnóstica, cuando posibilita la identificación de necesidades de formación, y de resultados, cuando permite la certificación de estas competencias (Sánchez, 2010a). En la literatura se encuentran diferentes proyectos e iniciativas para la evaluación de las competencias informacionales:

SAILS Standardized Assessment of Information Literacy Skills, ETS/ICT-Educational Testing Service/Information and Communications Technology, Bay Area Community College Information Competency Assessment Project, ISST-Information-Seeking Skills Test, TRAILS-Tool for Real-time Assessment of Information Literacy Skills, Information Skills Survey (ISS) for Assessment of Information Literacy inHigher Education, European Network on Information Literacy for a Culture of Information ENIL. (Sánchez, 2012: 55)

ALFIN-HUMASS es una herramienta diseñada específicamente para las titulaciones de humanidades y sociologías de la educación superior, donde su posición principal es la actitud.

\section{Metodología}

Esta investigación utiliza como instrumento de recolección de datos el cuestionario ALFIN-HUMASS. El estudio se limitó geográficamente a Cuba y su alcance se enfocó en la educación superior, específicamente en los estudian- 
tes de la titulación de Ciencias de la Información de la Universidad de la Habana.

La investigación se realizó en dos etapas. La primera consistió en la recopilación de datos durante el año académico 2011-2012 y en la segunda se realizó la exploración y análisis descriptivo de los datos en su conjunto. La muestra estuvo compuesta por 146 personas y predominan los estudiantes de primer año (Tabla 1).

Tabla 1. Participantes en el estudio

\begin{tabular}{|c|c|}
\hline Año académico & Cantidad de estudiantes encuestados \\
\hline Primer año & 55 \\
\hline Segundo año & 39 \\
\hline Tercer año & 29 \\
\hline Cuarto año & 23 \\
\hline Total & 146 \\
\hline
\end{tabular}

El cuestionario se aplicó de manera impresa en las aulas de la Facultad de Comunicación durante tres días consecutivos. En cada grupo de estudiantes se hizo una breve presentación de la encuesta y la explicación de los objetivos de ALFIN-HUMASS, con el objetivo de familiarizar a la población encuestada con el contenido de la herramienta. Las instrucciones fueron dadas por una misma investigadora para evitar los sesgos. Las respuestas fueron totalmente anónimas.

El ALFIN-HUMASS ${ }^{1}$ comprende 26 variables o ítems (Pinto y Lopes, 2010) que se agrupan en cuatro categorías o grandes competencias transversales:

1. Búsqueda (variables o ítems 1-8): 1. Saber utilizar fuentes de información impresa (ej. libros...). 2. Saber acceder y usar los catálogos automatizados. 3. Saber consultar y usar fuentes electrónicas de información primaria (ej. revistas...). 4. Saber utilizar fuentes electrónicas de información secundaria (ej. bases de datos...). 5. Conocer la terminología de tu materia. 6. Saber buscar y recuperar información en Internet (ej. búsquedas avanzadas, directorios, portales...). 7. Saber utilizar fuentes electrónicas informales de información (ej. blogs, listas de distribución...). 8. Conocer las estrategias de búsqueda de información (ej. descriptores, operadores booleanos...).

1 El instrumento ALFIN-HUMASS puede ser consultado de manera virtual en http://www.mariapinto.es/alfin-humass/ 
2. Evaluación (variables o ítems 9-13): 9. Saber evaluar la calidad de los recursos de información. 10. Reconocer en el texto las ideas del autor. 11. Conocer las fuentes de información científica (ej. tesis doctorales, actas de congresos...). 12. Ser capaz de determinar si la información que contiene un recurso está actualizada. 13. Conocer los autores o instituciones más relevantes en tu ámbito temático.

3. Tratamiento (variables o ítems 14-19): 14. Saber resumir y esquematizar la información. 15. Ser capaz de reconocer la estructuración de un texto. 16. Saber usar gestores de bases de datos (ej. Access, MySQL...). 17. Saber utilizar gestores de referencias bibliográficas (ej. Endnote, Reference Manager...). 18. Saber manejar programas estadísticos y hojas de cálculo (Ej. SPSS, Excel...). 19. Saber instalar programas informáticos.

4. Comunicación (variables o ítems 20-26): 20. Saber comunicar en público. 21. Saber comunicar en otros idiomas. 22. Saber redactar un documento (ej. informe, trabajo académico...). 23. Conocer el código ético de tu ámbito académico/profesional. 24. Conocer la legislación sobre el uso de la información y de la propiedad intelectual. 25. Saber hacer presentaciones académicas (ej. PowerPoint...). 26. Saber difundir la información en Internet (ej. webs, blogs, redes sociales...).

Cada una de estas variables o ítems es enmarcada en tres dimensiones:

1. Motivación o importancia (subjetiva y cuantitativa).

2. Autoeficacia o nivel de adquisición (subjetiva y cuantitativa).

3. Fuentes, vías o hábitos de aprendizaje (cualitativa).

En las dimensiones cuantitativas los estudiantes debían responder mediante una escala de Likert del 1 al 9, donde 1 era baja y 9 era alta. En la cualitativa, en cada variable o ítem señalaban una de las opciones que se les proponía: clase tradicional, biblioteca, de forma individual, cursos especializados. Los datos se recopilaron en Microsoft Excel y el análisis se realizó mediante el programa informático SPSS 15.0. En las dimensiones subjetivas y cuantitativas (la motivación y autoeficacia) se determinó, tanto para las variables o ítems como para las categorías, la tendencia central por la media y la dispersión de los datos a través de la desviación estándar. Se detectaron los valores extremos y atípicos con el fin de descubrir los puntos fuertes y los puntos débiles.

Dado el número y la diversidad de los estudiantes involucrados las comparaciones se realizaron de acuerdo al grado académico de los participantes; 
además, mediante la frecuencia se propone identificar las principales fuentes de aprendizaje utilizadas por la población encuestada.

Con respecto a la confiabilidad del instrumento se utiliza el coeficiente alfa de Cronbach. La consistencia interna alcanzada fue bastante alta (Tabla 2) y se puede decir que la encuesta es confiable. Las variaciones en las respuestas se deben a diferencias reales de opiniones, no porque el instrumento origine confusión.

Tabla 2. Análisis de la confiabilidad del instrumento (traducción de los resultados en inglés arrojados por el SPSS 15.0)

\begin{tabular}{|l|l|}
\hline \multicolumn{2}{|c|}{ Análisis de confiabilidad-Escala (Alpha) } \\
\hline Coeficiente de confiabilidad & Número de ítems: 52 \\
\hline Número de casos: 146,0 & \\
\hline Alpha: 9369 & \\
\hline
\end{tabular}

La validez externa de la encuesta de ALFIN-HUMASS es claramente apreciable. Sus elementos, las escalas y los procedimientos son generalizables y aplicables a la población en general, así como transferibles y aplicables a otro contexto similar. De un total de 146 casos el 100 \% fue validado.

\section{RESULTADOS Y DisCUSIÓN}

Los resultados que aparecen en la última fila de la Tabla 3 muestran una media global más alta en la dimensión de motivación (8.26) que en la autoeficacia (6.31); la diferencia entre estas medias globales fue de 1.95. En contraste, la desviación estándar fue más baja en la dimensión de la motivación (1.524) que en la autoeficacia (2.318). Se observa que las puntuaciones medias fueron mayores y más concentradas al evaluar la motivación, e inferiores y más dispersas al considerar la autoeficacia reconocida por los estudiantes.

Puede plantearse de manera general que existen 10 variables o ítems que logran mayor motivación y autoeficacia en los estudiantes al tener los valores más altos de las medias así como los valores más bajos de sus desviaciones (Tabla 3):

1. Saber utilizar fuentes de información impresa (ej. libros...).

3. Saber consultar y usar fuentes electrónicas de información primaria (ej. revistas...).

5. Conocer la terminología de tu materia.

9. Saber evaluar la calidad de los recursos de información.

10. Reconocer en el texto las ideas del autor. 
12. Ser capaz de determinar si la información que contiene un recurso está actualizada.

14. Saber resumir y esquematizar la información.

20. Saber comunicar en público.

22. Saber redactar un documento (ej. informe, trabajo académico...).

25. Saber hacer presentaciones académicas (ej. PowerPoint...).

La variable 22 (Saber redactar un documento) y la 25 (Saber hacer presentaciones académicas) son las que más se destacan de manera positiva, porque se encuentran tanto dentro de los cinco primeros valores más altos de las medias como también dentro de los cinco valores más bajos de sus desviaciones. Esta observación puede aplicarse tanto a las dimensiones de la motivación como de la autoeficacia. Asimismo, de manera general existen 9 variables o ítems que tienen los valores más bajos de las medias así como los valores más altos de sus desviaciones, tanto en la motivación como en la autoeficacia (Tabla 3):

4. Saber utilizar fuentes electrónicas de información secundaria (ej. bases de datos...).

7. Saber utilizar fuentes electrónicas informales de información (ej. blogs, listas de distribución...).

8. Conocer las estrategias de búsqueda de información (ej. descriptores, operadores booleanos...).

16. Saber usar gestores de bases de datos (ej. Access, MySQL...).

17. Saber utilizar gestores de referencias bibliográficas (ej. Endnote, Reference Manager...).

18. Saber manejar programas estadísticos y hojas de cálculo (ej. SPSS, Excel...).

19. Saber instalar programas informáticos.

24. Conocer la legislación sobre el uso de la información y de la propiedad intelectual.

26. Saber difundir la información en Internet (ej. webs, blogs, redes sociales...)

La variable 17 (Saber utilizar gestores de referencias bibliográficas) es la más negativa, porque se encuentra dentro de los cinco valores más bajos de las medias y dentro de los cinco valores más altos de sus desviaciones. Esta observación puede aplicarse tanto a las dimensiones de la motivación como de la autoeficacia, es decir, esta variable es la que consideran menos importante y peor adquirida. 
Tabla 3. Medias y desviaciones estándar de las variable o ítems en $\mathrm{N}=146$

\begin{tabular}{|c|c|c|c|c|c|}
\hline & \multirow[t]{2}{*}{ Variables o ítems } & \multicolumn{2}{|c|}{ Motivación } & \multicolumn{2}{|c|}{ Autoeficacia } \\
\hline & & Media & $\begin{array}{l}\text { Desviación } \\
\text { estándar }\end{array}$ & Media & $\begin{array}{l}\text { Desviación } \\
\text { estándar }\end{array}$ \\
\hline 1. & $\begin{array}{l}\text { Saber utilizar fuentes de información } \\
\text { impresa (ej. libros...). }\end{array}$ & 8.39 & 1.285 & 7.55 & 1.799 \\
\hline 2. & $\begin{array}{l}\text { Saber acceder y usar los catálogos } \\
\text { automatizados. }\end{array}$ & 8.13 & 1.472 & 5.73 & 2.431 \\
\hline 3. & $\begin{array}{l}\text { Saber consultar y usar fuentes electrónicas } \\
\text { de información primaria (ej. revistas...). }\end{array}$ & 8.41 & 1.354 & 6.88 & 2.144 \\
\hline 4. & $\begin{array}{l}\text { Saber utilizar fuentes electrónicas de infor- } \\
\text { mación secundaria (ej. bases de datos...). }\end{array}$ & 8.12 & 1.686 & 6.24 & 2.371 \\
\hline 5. & Conocer la terminología de tu materia. & 8.60 & 1.214 & 6.89 & 1.799 \\
\hline 6. & $\begin{array}{l}\text { Saber buscar y recuperar información en } \\
\text { Internet (ej. búsquedas avanzadas, directo- } \\
\text { rios, portales...). }\end{array}$ & 8.42 & 1.579 & 6.34 & 2.544 \\
\hline 7. & $\begin{array}{l}\text { Saber utilizar fuentes electrónicas infor- } \\
\text { males de información (ej. blogs, listas de } \\
\text { distribución...). }\end{array}$ & 7.66 & 1.882 & 5.48 & 2.570 \\
\hline 8. & $\begin{array}{l}\text { Conocer las estrategias de búsqueda de } \\
\text { información (ej. descriptores, operadores } \\
\text { booleanos...). }\end{array}$ & 8.04 & 1.890 & 5.44 & 2.966 \\
\hline 9. & $\begin{array}{l}\text { Saber evaluar la calidad de los recursos de } \\
\text { información. }\end{array}$ & 8.38 & 1.430 & 6.35 & 2.033 \\
\hline 10. & Reconocer en el texto las ideas del autor. & 8.30 & 1.367 & 7.39 & 1.837 \\
\hline 11. & $\begin{array}{l}\text { Conocer la tipología de las fuentes de } \\
\text { información científica (ej. tesis doctorales, } \\
\text { actas de congresos...). }\end{array}$ & 8.29 & 1.172 & 7.28 & 1.975 \\
\hline 12. & $\begin{array}{l}\text { Ser capaz de determinar si la información } \\
\text { que contiene un recurso está actualizada. }\end{array}$ & 8.44 & 1.245 & 6.53 & 2.088 \\
\hline 13. & $\begin{array}{l}\text { Conocer los autores o instituciones más } \\
\text { relevantes en tu ámbito temático. }\end{array}$ & 8.35 & 1.625 & 6.22 & 2.389 \\
\hline 14. & $\begin{array}{l}\text { Saber resumir y esquematizar la informa- } \\
\text { ción. }\end{array}$ & 8.40 & 1.465 & 6.82 & 2.126 \\
\hline 15. & $\begin{array}{l}\text { Ser capaz de reconocer la estructuración } \\
\text { de un texto. }\end{array}$ & 8.01 & 1.686 & 6.65 & 2.138 \\
\hline 16. & $\begin{array}{l}\text { Saber usar gestores de bases de datos (ej. } \\
\text { Access, MySQL...). }\end{array}$ & 8.10 & 1.604 & 5.03 & 2.613 \\
\hline 17. & $\begin{array}{l}\text { Saber utilizar gestores de referencias } \\
\text { bibliográficas (ej. Endnote, Reference } \\
\text { Manager...). }\end{array}$ & 7.99 & 1.974 & 5.35 & 2.954 \\
\hline 18. & $\begin{array}{l}\text { Saber manejar programas estadísticos y } \\
\text { hojas de cálculo (ej. SPSS, Excel...). }\end{array}$ & 8.01 & 1.738 & 5.91 & 2.682 \\
\hline 19. & Saber instalar programas informáticos. & 7.86 & 2.019 & 5.88 & 3.022 \\
\hline 20. & Saber comunicar en público. & 8.57 & 1.250 & 6.93 & 1.934 \\
\hline 21. & Saber comunicar en otros idiomas. & 8.45 & 1.391 & 4.95 & 2.597 \\
\hline
\end{tabular}




\begin{tabular}{|c|l|c|c|c|c|}
\hline 22. & $\begin{array}{l}\text { Saber redactar un documento (ej. informe, } \\
\text { trabajo académico...). }\end{array}$ & 8.71 & 0.868 & 7.31 & 1.678 \\
\hline 23. & $\begin{array}{l}\text { Conocer el código ético de tu ámbito } \\
\text { académico/profesional. }\end{array}$ & 8.24 & 1.838 & 6.64 & 2.293 \\
\hline 24. & $\begin{array}{l}\text { Conocer la legislación sobre el uso de la } \\
\text { información y de la propiedad intelectual. }\end{array}$ & 8.07 & 1.933 & 5.30 & 2.547 \\
\hline 25. & $\begin{array}{l}\text { Saber hacer presentaciones académicas } \\
\text { (ej. PowerPoint...). }\end{array}$ & 8.59 & 0.809 & 7.83 & 1.734 \\
\hline 26. & $\begin{array}{l}\text { Saber difundir la información en Internet } \\
\text { (ej. webs, blogs, redes sociales...). }\end{array}$ & 8.22 & 1.861 & 5.21 & 3.010 \\
\hline & & 8.26 & 1.524 & 6.31 & 2.318 \\
\hline
\end{tabular}

La relación entre la motivación y la autoeficacia mediante el análisis de pares demostró que las diferencias entre las medias son significativas, ubicándose entre los valores 1 y 3 (Tabla 4). Puntuaciones por debajo de 1 se observan en las variables 1 (Saber utilizar fuentes de información impresa), 10 (Reconocer en el texto las ideas del autor) y 25 (Saber hacer presentaciones académicas). En ellas tanto la motivación como la autoeficacia tienen altos valores. Puntuaciones por encima de 3 se observan en los ítems 16 (Saber usar gestores de bases de datos) y 26 (Saber difundir la información en Internet), es decir, el valor de la motivación es alto y el de la autoeficacia es bajo, hay espacio para la mejora.

Los puntajes más altos de las desviaciones estándar de las diferencias reflejan menores niveles de formación y grandes oportunidades de mejora para una mayor formación. La mayoría se encuentran por encima de 2, las oportunidades de mejora para la población encuestada se centran en este conjunto de variables o ítems (Tabla 4).

A partir del coeficiente de Pearson ( $r$ ) que se muestra en la Tabla 4 se puede enfatizar que existe correlación entre la motivación y la autoeficacia. Es decir, cada vez que aumenta la motivación la autoeficacia también lo hace. Puede plantearse entonces que cuanto más nivel creen que se tiene mayor importancia le dan a esa competencia, es probable que cuanto más se entrene al estudiante en una competencia determinada y mayor nivel vaya adquiriendo en ella, mayor será la importancia y el valor que otorgue a la misma.

Tomando en cuenta la $r^{2}$ (coeficiente de Pearson al cuadrado, determina en qué medida la motivación contribuye a la autoeficacia en las variables o ítems -correlación entre las dimensiones motivación y autoeficacia-) en la Tabla 4, la motivación en los puntos 10. Reconocer en el texto las ideas del autor y 23. Conocer el código ético de tu ámbito académico/profesional contribuye al $20 \%$ de su autoeficacia. Existen 3 variables o ítems en los que la motivación contribuye al $10 \%$ de la autoeficacia. 
Tabla 4. Diferencias entre la motivación y la autoeficacia

\begin{tabular}{|c|c|c|c|c|c|}
\hline & Variables o ítems & \begin{tabular}{|c|} 
Diferencias entre \\
las medias
\end{tabular} & \begin{tabular}{|c|} 
Diferencias de \\
las desviaciones \\
estándar
\end{tabular} & $\begin{array}{l}\text { Coeficiente de } \\
\text { Pearson } r\end{array}$ & $r^{2}$ \\
\hline 1. & $\begin{array}{l}\text { Saber utilizar fuentes de información } \\
\text { impresa (ej. libros...). }\end{array}$ & 0.84 & 1.8581 & 0.31 & 0.10 \\
\hline 2. & $\begin{array}{l}\text { Saber acceder y usar los catálogos } \\
\text { automatizados. }\end{array}$ & 2.4 & 2.5633 & 0.21 & 0.04 \\
\hline 3. & $\begin{array}{l}\text { Saber consultar y usar fuentes } \\
\text { electrónicas de información primaria } \\
\text { (ej. revistas...). }\end{array}$ & 1.53 & 2.0882 & 0.36 & 0.13 \\
\hline 4. & $\begin{array}{l}\text { Saber utilizar fuentes electrónicas de } \\
\text { información secundaria (ej. bases de } \\
\text { datos...). }\end{array}$ & 1.88 & 2.4576 & 0.30 & 0.09 \\
\hline 5. & $\begin{array}{l}\text { Conocer la terminología de tu } \\
\text { materia. }\end{array}$ & 1.71 & 1.7210 & 0.40 & 0.16 \\
\hline 6. & $\begin{array}{l}\text { Saber buscar y recuperar infor- } \\
\text { mación en Internet (ej. búsquedas } \\
\text { avanzadas, directorios, portales...). }\end{array}$ & 2.08 & 2.5388 & 0.31 & 0.10 \\
\hline 7. & $\begin{array}{l}\text { Saber utilizar fuentes electrónicas } \\
\text { informales de información (ej. blogs, } \\
\text { listas de distribución...). }\end{array}$ & 2.18 & 2.8042 & 0.24 & 0.06 \\
\hline 8. & $\begin{array}{l}\text { Conocer las estrategias de búsqueda } \\
\text { de información (ej. descriptores, } \\
\text { operadores booleanos...). }\end{array}$ & 2.6 & 2.9062 & 0.35 & 0.12 \\
\hline 9. & $\begin{array}{l}\text { Saber evaluar la calidad de los } \\
\text { recursos de información. }\end{array}$ & 2.03 & 2.1972 & 0.23 & 0.05 \\
\hline 10. & $\begin{array}{l}\text { Reconocer en el texto las ideas del } \\
\text { autor. }\end{array}$ & 0.91 & 1.7396 & 0.44 & 0.20 \\
\hline 11. & $\begin{array}{l}\text { Conocer la tipología de las fuentes } \\
\text { de información científica (ej. tesis } \\
\text { doctorales, actas de congresos...). }\end{array}$ & 1.01 & 2.0772 & 0.21 & 0.04 \\
\hline 12. & $\begin{array}{l}\text { Ser capaz de determinar si la infor- } \\
\text { mación que contiene un recurso está } \\
\text { actualizada. }\end{array}$ & 1.91 & 2.2019 & 0.20 & 0.04 \\
\hline 13. & $\begin{array}{l}\text { Conocer los autores o institucio- } \\
\text { nes más relevantes en tu ámbito } \\
\text { temático. }\end{array}$ & 2.13 & 2.2733 & 0.41 & 0.17 \\
\hline 14. & $\begin{array}{l}\text { Saber resumir y esquematizar la } \\
\text { información. }\end{array}$ & 1.58 & 2.1608 & 0.32 & 0.10 \\
\hline 15. & $\begin{array}{l}\text { Ser capaz de reconocer la estructu- } \\
\text { ración de un texto. }\end{array}$ & 1.36 & 2.1191 & 0.41 & 0.16 \\
\hline 16. & $\begin{array}{l}\text { Saber usar gestores de bases de } \\
\text { datos (ej. Access, MySQL...). }\end{array}$ & 3.07 & 2.9299 & 0.10 & 0.01 \\
\hline 17. & $\begin{array}{l}\text { Saber utilizar gestores de referencias } \\
\text { bibliográficas (ej. Endnote, Reference } \\
\text { Manager...). }\end{array}$ & 2.64 & 2.9976 & 0.31 & 0.10 \\
\hline 18. & $\begin{array}{l}\text { Saber manejar programas estadís- } \\
\text { ticos y hojas de cálculo (ej. SPSS, } \\
\text { Excel...). }\end{array}$ & 2.1 & 2.8049 & 0.25 & 0.06 \\
\hline
\end{tabular}




\begin{tabular}{|l|l|c|c|c|c|}
\hline 19. & $\begin{array}{l}\text { Saber instalar programas informá- } \\
\text { ticos. }\end{array}$ & 1.98 & 3.0215 & 0.33 & 0.11 \\
\hline 20. & Saber comunicar en público. & 1.64 & 2.2719 & 0.03 & 0.00 \\
\hline 21. & Saber comunicar en otros idiomas. & 3.5 & 2.6929 & 0.20 & 0.04 \\
\hline 22. & $\begin{array}{l}\text { Saber redactar un documento (ej. } \\
\text { informe, trabajo académico...). }\end{array}$ & 1.4 & 1.6911 & 0.24 & 0.06 \\
\hline 23. & $\begin{array}{l}\text { Conocer el código ético de tu ámbito } \\
\text { académico/profesional. }\end{array}$ & 1.6 & 2.2132 & 0.44 & 0.20 \\
\hline 24. & $\begin{array}{l}\text { Conocer la legislación sobre el uso } \\
\text { de la información y de la propiedad } \\
\text { intelectual. }\end{array}$ & 2.77 & 2.7038 & 0.28 & 0.08 \\
\hline 25. & $\begin{array}{l}\text { Saber hacer presentaciones acadé- } \\
\text { micas (ej. Power Point...). }\end{array}$ & 0.76 & 1.7878 & 0.17 & 0.03 \\
\hline 26. & $\begin{array}{l}\text { Saber difundir la información en } \\
\text { Internet (ej. webs, blogs, redes } \\
\text { sociales...). }\end{array}$ & 3.01 & 3.2266 & 0.19 & 0.04 \\
\hline
\end{tabular}

Al realizar un análisis descriptivo de las categorías o grandes competencias transversales consideradas por el ALFIN-HUMASS se puede plantear que las cuatro presentaron un resultado diferente (Tabla 5). En la alta motivación sobresale la categoría de comunicación (8.41), de lo que se asume que esta competencia transversal es la más importante para el estudiantado. En cuanto a la autoeficacia, la categoría de evaluación fue la que obtuvo el valor más alto (6.75), de forma que esta competencia transversal es la mejor adquirida, es decir, en la que más formados se perciben. Las puntuaciones de la categoría de tratamiento fueron inferiores a la media tanto en la motivación como en la autoeficacia; esta es la categoría en la que menor nivel de adquisición perciben y es a la que menos importancia conceden. La categoría que creen menos importante es el factor "[...] que menos les preocupa en el sentido de que es al que menos interés prestan a la hora de formarse o mejorar en su nivel de competencia" (Pinto y Puertas, 2012: 9).

Tabla 5. Tendencia Central de Categorías N=146

\begin{tabular}{|l|l|c|c|c|c|}
\hline \multicolumn{2}{|c|}{} & \multicolumn{2}{|c|}{ Motivación } & \multicolumn{2}{c|}{ Autoeficacia } \\
\hline & $\begin{array}{c}\text { Categorías o grandes } \\
\text { competencias transversales }\end{array}$ & Media & $\begin{array}{c}\text { Desviación } \\
\text { Estándar }\end{array}$ & Media & $\begin{array}{c}\text { Desviación } \\
\text { estándar }\end{array}$ \\
\hline 1 & Búsqueda & 8.22 & 1.54 & 6.32 & 2.33 \\
\hline 2 & Evaluación & 8.35 & 1.37 & 6.75 & 2.06 \\
\hline 3 & Tratamiento & 8.06 & 1.75 & 5.94 & 2.59 \\
\hline 4 & Comunicación & 8.41 & 1.42 & 6.31 & 2.26 \\
\hline
\end{tabular}

Nótese que en la categoría Tratamiento la variable o ítem que consideran más importante es a su vez en la que se perciben mejor preparados (Tabla 6). 
Tabla 6. Análisis por categorías

\begin{tabular}{|c|c|c|c|c|}
\hline Categorías & $\begin{array}{l}\text { Competencia } \\
\text { más importante }\end{array}$ & $\begin{array}{c}\text { Competencia } \\
\text { menos importante }\end{array}$ & $\begin{array}{c}\text { Competencia } \\
\text { mejor adquirida }\end{array}$ & $\begin{array}{c}\text { Competencia } \\
\text { peor adquirida }\end{array}$ \\
\hline $\begin{array}{l}\text { Búsqueda de } \\
\text { información }\end{array}$ & $\begin{array}{l}\text { Conocer la termino- } \\
\text { logía de tu materia } \\
8.60\end{array}$ & $\begin{array}{l}\text { Saber utilizar fuentes } \\
\text { electrónicas informa- } \\
\text { les de información } \\
7.66\end{array}$ & $\begin{array}{l}\text { Saber utilizar fuentes } \\
\text { de información } \\
\text { impresa } \\
7.55\end{array}$ & $\begin{array}{l}\text { Conocer las estrate- } \\
\text { gias de búsqueda de } \\
\text { información } \\
5.44\end{array}$ \\
\hline $\begin{array}{l}\text { Evaluación de } \\
\text { la información } \\
\text { Medias }\end{array}$ & $\begin{array}{l}\text { Ser capaz de deter- } \\
\text { minar si la informa- } \\
\text { ción de un recurso } \\
\text { está actualizada } \\
8.44\end{array}$ & $\begin{array}{l}\text { Conocer la tipología } \\
\text { de las fuentes de in- } \\
\text { formación científica } \\
8.29\end{array}$ & $\begin{array}{l}\text { Reconocer en el } \\
\text { texto las ideas del } \\
\text { autor } \\
7.39\end{array}$ & $\begin{array}{l}\text { Conocer los autores } \\
0 \text { instituciones }\end{array}$ \\
\hline $\begin{array}{l}\text { Tratamiento de } \\
\text { la información } \\
\text { Medias }\end{array}$ & 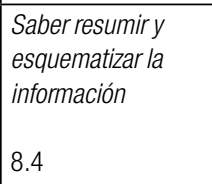 & $\begin{array}{l}\text { Saber instalar pro- } \\
\text { gramas informáticos } \\
7.86\end{array}$ & $\begin{array}{l}\begin{array}{l}\text { Saber resumiry } \\
\text { esquematizar la } \\
\text { información }\end{array} \\
6.82\end{array}$ & $\begin{array}{l}\text { Saber utilizar ges- } \\
\text { tores de referencias } \\
\text { bibliográficas } \\
5.03\end{array}$ \\
\hline $\begin{array}{l}\text { Comunicación } \\
\text { y difusión de la } \\
\text { información }\end{array}$ & $\begin{array}{l}\text { Saber redactar un } \\
\text { documento }\end{array}$ & $\begin{array}{l}\text { Conocer la legisla- } \\
\text { ción sobre el uso de } \\
\text { la información y de la } \\
\text { propiedad intelectual } \\
8.07\end{array}$ & $\begin{array}{l}\text { Saber hacer presen- } \\
\text { taciones académicas } \\
7.83\end{array}$ & $\begin{array}{l}\text { Saber comunicar en } \\
\text { otros idiomas }\end{array}$ \\
\hline
\end{tabular}

En cuanto a los años académicos (Tabla 7), la mayor motivación se encuentra en los alumnos de cuarto año. Es preciso trabajar con los estudiantes de primer año de manera intensa pues tienen baja motivación y baja eficacia. La mayor eficacia se encuentra en los de segundo año.

Tabla 7. Tendencia central por grado académico

\begin{tabular}{|l|c|c|c|c|}
\hline Año académico & \multicolumn{2}{|c|}{ Motivación } & \multicolumn{2}{c|}{ Autoeficacia } \\
\hline & Media & Desviación estándar & Media & Desviación estándar \\
\hline Primer año & 8.07 & 1.84 & 5.33 & 2.72 \\
\hline Segundo año & 8.45 & 1.38 & 6.96 & 2.15 \\
\hline Tercer año & 8.17 & 1.53 & 6.79 & 1.99 \\
\hline Cuarto año & 8.51 & 1.06 & 6.92 & 2.21 \\
\hline
\end{tabular}

Un análisis de las frecuencias en la tercera dimensión (hábitos de aprendizaje) de las variables o ítems también proporciona información relevante para la investigación. La Figura 1 muestra las proporciones de las cuatro categorías principales de hábitos de aprendizaje: el autoaprendizaje, clases, biblioteca y cursos. El autoaprendizaje y las clases fueron particularmente sig- 
nificativas entre la población encuestada; sin embargo, la biblioteca, considerada como un hábito de aprendizaje, es mucho menos relevante.

\section{Hábitos de aprendizaje}

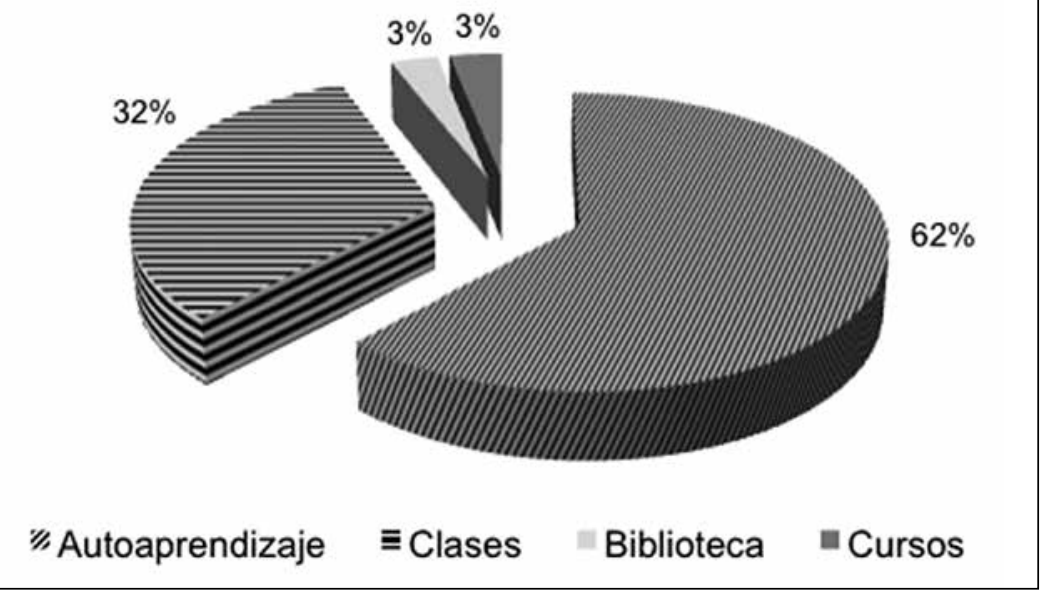

Figura 1. Hábitos de aprendizaje

\section{Conclusiones}

La investigación evidencia que las competencias informacionales son un tema importante para la titulación de Ciencias de la Información. En general los estudiantes de CI reconocieron niveles altos de motivación para la mayoría de las variables o ítems, en tanto que "[...] la motivación es la piedra angular de la alfabetización informacional” (Pinto, 2011: 146); sin embargo, sus niveles de autoeficacia son significativamente bajos. Esta oportunidad debe aprovecharse para actuar sobre la base de la motivación existente en la población encuestada y contribuir a elevar los niveles de autoeficacia.

En cuanto a las variables o ítems que mostraron niveles altos tanto en la motivación como en la autoeficacia, sería conveniente continuar este estudio evidenciando la relación entre las creencias y la verdadera satisfacción. No obstante, estas variables fuertes deben ser aprovechadas por la facultad, convocando a los estudiantes a que actúen como facilitadores del desarrollo de las mismas en otras titulaciones, haciendo un llamado a que asuman nuevos roles en diferentes espacios universitarios. 
El estudiantado se encuentra menos motivado en la competencia relacionada con el tratamiento de la información, en este caso hay que concienciar sobre la importancia de ésta en el profesional de la información. Además, se pone de manifiesto que la competencia con menos motivación es aquella en la que los estudiantes se sienten menos preparados y este resultado llama la atención puesto que en el plan de estudios reciben diferentes asignaturas que responden a esta competencia.

Es necesario elevar prioritariamente los niveles de motivación en las variables 2 . Saber acceder y usar los catálogos automatizados, 11 . Conocer la tipología de las fuentes de información científica, 15. Ser capaz de reconocer la estructura de un texto y 23. Conocer el código ético de tu ámbito académico/ profesional. Los profesionales de la información están comprometidos con la excelencia en el uso de las nuevas tecnologías de información y comunicación, en el manejo de la información más allá de las fuentes tradicionales, en la identificación del contenido de la información, en la ética profesional, así como con los valores y principios de la profesión. En este sentido, se precisan acciones inmediatas para mejorar la motivación y la autoeficacia en la variable relacionada con el uso de los gestores de las referencias bibliográficas, pues presenta bajos valores aun cuando es imprescindible para los futuros formadores de competencias informacionales.

Llama la atención que el ítem que presenta los mayores valores tanto en la motivación como en la autoeficacia en este trabajo, así como el que presenta los menores coinciden con los resultados obtenidos por Pinto (2011) y Pinto y Puertas (2009 y 2012). También es importante la dispersión de los datos, que reflejan la falta de uniformidad y coherencia entre la población encuestada con respecto a competencias informacionales, aun cuando son estudiantes de la especialidad de CI.

En cuanto a las categorías de competencias informacionales analizadas, cada variable o ítem debe ser objeto de desarrollo como parte de la formación reglada, es decir, es preciso integrar programas de ALFIN en el currículo de formación de Ciencias de la Información. Evidente es la necesidad de lograr una autoeficacia alta en estos estudiantes porque son ellos los que deben marcar la diferencia en la exigencia que conlleva la sociedad de la información, sólo así llegarán a ser capaces de buscar desafíos y capitalizar nuevas oportunidades. Además, es este profesional el responsable del desarrollo de las competencias informacionales, de ahí que requiera una sólida preparación.

En el aspecto cualitativo de la investigación, el predominio del aprendizaje individual corrobora la necesidad de que el desarrollo de las competencias informacionales se integre en la práctica al plan de estudios de la espe- 
cialidad, además evidencia la necesidad de aumentar la participación de la biblioteca de la facultad y de la colaboración entre bibliotecarios y profesores. Esta integración debe empezar desde primer año con vistas a mejorar la motivación y la formación.

No cabe duda que dentro de la educación superior cubana éste es un estudio pionero que abre las puertas a nuevas investigaciones en el contexto latinoamericano, pues contar con un diagnóstico de las competencias informacionales desde la perspectiva del estudiante permite actuar de manera concreta y correcta.

\section{Agradecimientos}

A la profesora María Pinto Molina por facilitar la información relativa al cuestionario ALFIN-HUMASS e impulsar este trabajo.

A los profesores y estudiantes de CI de la Universidad de la Habana, especialmente a la dra. Gloria Ponjuán.

\section{BiBLIOGRAFÍA}

ACRL/ALA (2000), Normas sobre aptitudes para el acceso y uso de la información en la educación superior. Disponible en: http://www. aab.es/pdfs/baab60/60a6.pdf [Fecha de consulta: 21 de mayo de 2009].

Gómez-Hernández, J. A. (2009), "Aprender a enseñar competencias informacionales a los usuarios: avances en la formación profesional en España”, en Anuario ThinkEPI, 3, 106-113.

González, C. L.; Sánchez, Y. y Lezcano, Y. (2012), "Estudio exploratorio sobre las competencias informacionales de los estudiantes de la Universidad de la Habana”, en Ciencias de la Información, 43 (2), 61-68.

Meneses-Placeres, G. y Frías-Guzmán, M. (2011), “La alfabetización informacional en los procesos curriculares de las ciencias de la información en Cuba", en Revista Interamericana de Bibliotecología, 34 (1), 9-22.

Pinto, M. (2011), "An Approach to the Internal Facet of Information Literacy Using the IL-HUMASS Survey", en Journal of Academic Librarianship, 37 (2), 145-154.

(2010), "Design of the IL-HUMASS survey on information literacy in higher education: A self-assessment approach", en Journal of Information Science, 36 (86), 86-103.

— y Lopes, C. (2010), "IL-HUMASS - instrumento de avaliação de competências em literacia da informação", en X Congreso de la $B A D$, 7-9 de abril, Guimaraes, Portugal. 
Pinto, M. y Puertas, S. (2012), "Autoevaluación de la competencia informacional en los estudios de Psicología desde la percepción del estudiante”, en Anales de Documentación, 15 (2), 1-15.

y Puertas, S. (2009), "El aprendizaje por competencias transversales: la competencia informacional y comunicacional de los estudiantes de la titulación de Comunicación Audiovisual”, en Diálogos de la Comunicación, 78, 1-14.

—; Doucet, A. V. y Fernández-Ramos, A. (2008), "The role of information competencies and skills in learning to abstract", en Journal of Information Science, 34 (6), 799-815.

Quindemil, E. (2010), "Introducción de la alfabetización informacional en la especialidad de Bibliotecología y Ciencias de la Información”, en Acimed, 21 (2).

Sánchez, M. (2012), "La gestión de competencias informacionales en las universidades: reto para los profesionales de la información”, en Revista de Comunicación Vivat Academia, 121, 50-64. - (2010a), "Acotaciones teórico-conceptuales sobre la evaluación y desarrollo de las competencias desde la perspectiva informacional”, en Ciencias de la Información, 41 (2), 27-37.

(2010b), Competencias informacionales en la formación de las BioCiencias en Cuba, tesis doctoral, Universidad de Granada, España, Facultad de Comunicación y Documentación.

(2008a), "Breves comentarios teóricos sobre la evaluación, el desarrollo y la certificación de las competencias en información. A propósito de una revisión de la literatura”, en Acimed, 18 (1).

- (2008b), "Las competencias desde la perspectiva informacional: apuntes introductorios a nivel terminológico y conceptual, escenarios e iniciativas”, en Ciência da Informação, 37 (1), 107-120.

- y Vega, J. C. (2004), "El profesional de la información en el ámbito iberoamericano”, en ACIMED, 12. 